\title{
Evaluation of models of homologue search with respect to their efficiency on meiotic pairing
}

\author{
JOSEF LOIDL \& HELMUT LÄNGER* \\ Institute of Botany, University of Vienna, Rennweg 14, A-1030 Vienna, Austria, and *Institute of Algebra and Discrete \\ Mathematics, Technical University of Vienna, Wiedner Hauptstraße 8-10, A-1040 Vienna, Austria
}

\begin{abstract}
We have constructed models of how homologous chromosomes come together at meiotic prophase for pairing and recombination. For these models we have calculated how many homology testing events are necessary on average to ensure that each chromosome finds its respective partner. We have identified several conditions that would greatly reduce the effort spent on homology search when compared with a search strategy which is based on fortuitous homologous contacts. The models are discussed in the light of experimental evidence.
\end{abstract}

Keywords: chromosome pairing, homology, meiotic prophase, synaptonemal complex.

\section{Introduction}

There are opposite views as to whether chromosome pairing at meiosis is a random process based on chance encounter of homologues or whether there exist conditions which ensure a straightforward alignment of homologues or, at least, reduce randomness to some extent (for review see Loidl, 1990).

Critics of a purely random homologue search model argue rather intuitively that the likelihood of the finding of homologues within the limited period of meiotic prophase might be too low. Here we have mathematically evaluated the expenditure on homology finding in terms of the number of testing events required, by various hypothetical search strategies which depend on random processes to different degrees. We will discuss how reduction of time and chromosome movements may be conferred by various putative mechanisms of controlling homologue encounter.

The models introduced here are based on the assumption that homologue recognition is executed by molecular processes that are exerted at distinct individual loci within chromosomes, which will be termed

\footnotetext{
${ }^{1}$ Current controversial hypotheses on the initiation of crossovers preceding vs. following the formation of a synaptonemal complex are equally satisfied by the models.
}

'recognition sites' (for discussion see Loidl, 1990). This term will be used for any DNA sequence or chromosome region or other unit that autonomously can perform a molecular match ('testing event') to initiate a crossover directly or to establish homology for subsequent synapsis ${ }^{1}$. According to a current hypothesis, a testing event can be conceived as the matching of single stranded DNA molecules in transient heteroduplexes which might form near double strand breaks (see, e.g. Smithies \& Powers, 1986; Carpenter, 1987; Sun et al., 1989; Lichten et al., 1990; Padmore et al., 1991).

In the following, various models for homologue search will be described and formulae will be provided for their relative pairing efficiencies. Pairing efficiencies will be expressed in terms of the (mean) number of testing events $(t)$ which are required to associate a given number of corresponding recognition sites $(2 r)$ in homologous pairs. In most organisms, recognition sites may constitute autonomous pairing units of less than chromosome size. For those cases where one recognition site per chromosome suffices for the identification of a homologous chromosome pair, $2 r$ equals the diploid chromosome number $2 n$. However, for comparing relative efficiencies of the models, the number of independently acting recognition sites per chromosome is of no relevance. 


\section{Models and formulae (Derivations of formulae are given in Appendix I)}

\section{Random search of the whole diploid genome}

Here chromosomes meet by chance and test homology (Fig. 1). If homology is not found, the two chromosomes separate again and continue the search; if it is found, they establish a physical connection. There are two ways for continuing this process.

1a Homologous chromosomes that have found each other are committed to each other by some physical connection and yet are accessible as partners for homology testing for the others which are still searching. Thus, even late at pairing when most chromosomes have found their partner, all of them continue to get involved in homology tests as the testing machinery cannot discriminate between bound and free chromosomes. This random search strategy can be calculated to require a mean number of testing events

$t=r(2 r-1) \sum_{i=1}^{r} i^{-1}$

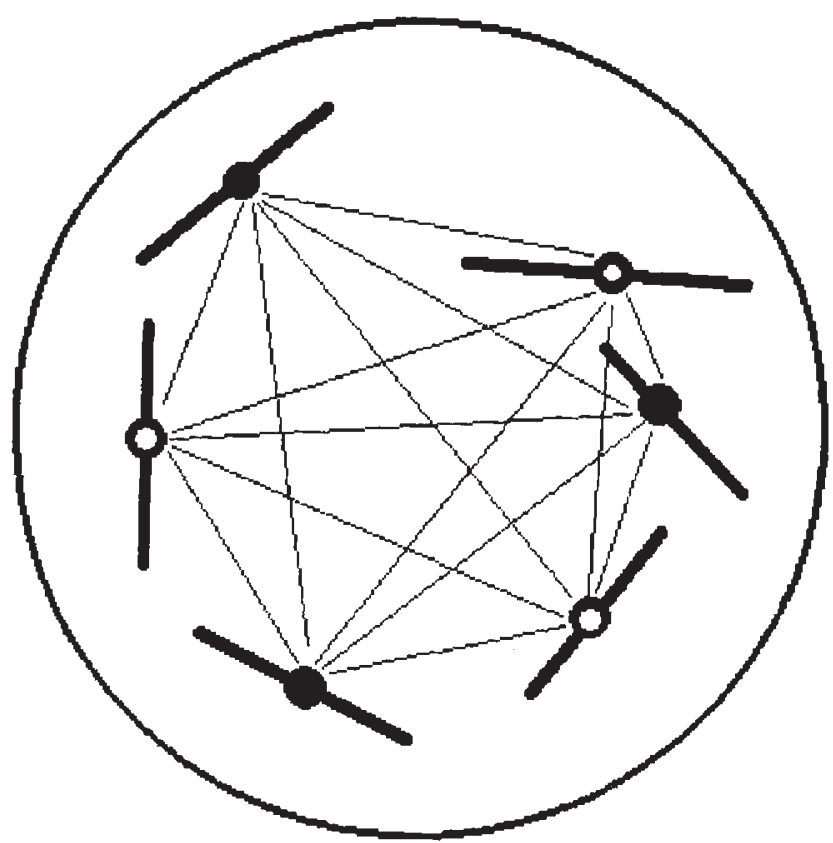

Fig. 1 Representation of the model of random search of the whole diploid genome. Three chromosomes of each parental set are drawn and their homology recognition sites are depicted as open or closed circles according to their parental origin. For the sake of simplicity each chromosome possesses only one homology recognition site. Lines between the chromosomes represent all possible different combinations for homology testing events.
For large $r$ this formula can be simplified to

$t \approx 2 r^{2} \log r$.

(Here and in the following, log denotes the natural logarithm (logarithm to base $e$ ). It holds

$\left|\log r-\sum_{i-1}^{r} i^{-1}\right|<1$

for $r>1$.)

1b Chromosomes that have found their partner cease to be involved in testing events. This improves the efficiency to a mean number of testing events

$t=r^{2}$.

\section{Intergenomic homologue search}

Here chromosomes do not screen the whole diploid set but each chromosome of one haploid set limits or preferentially extends its search for homology to chromosomes of the other set only (Fig. 2). It is conceivable that either by topological (genomic separation) or molecular criteria ('genomic imprinting') the two chromosome sets are distinct, which helps them to avoid, or at least to reduce, homologue search within one parental set. This process, too, could have two

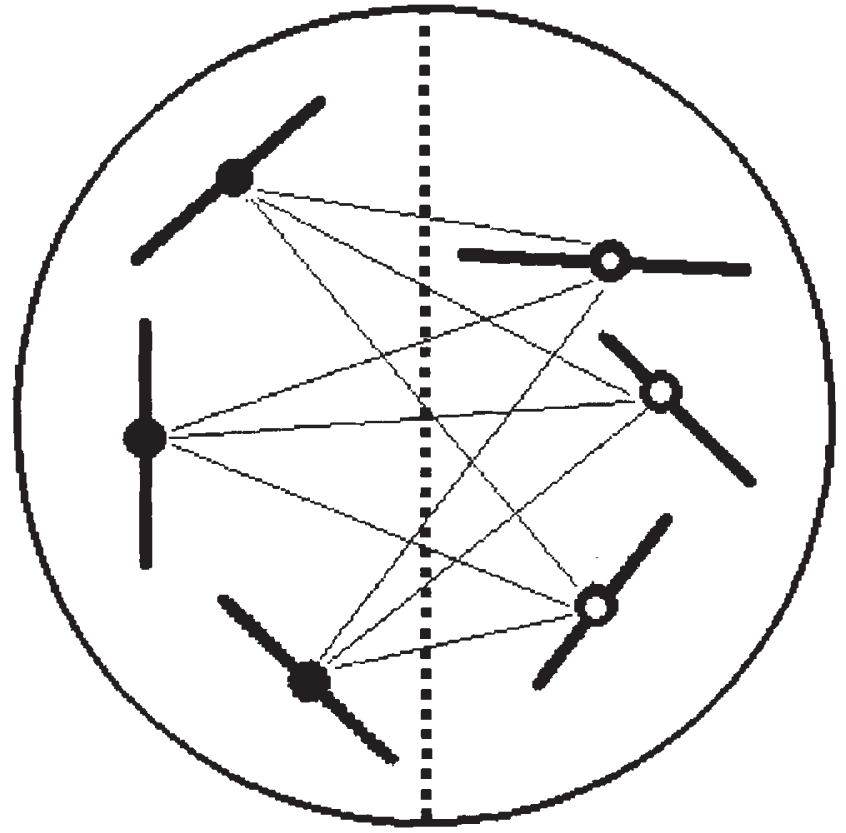

Fig. 2 Representation of the model of intergenomic homology search. Homology testing events take place only between members of different parental chromosome sets that might be separated spatially as indicated by broken line. For further explanations see legend to Fig. 1. 
variants differing by the behaviour of chromosomes that have found their partner.

2a They continue the search, with the mean number of testing events being

$t=r^{2} \sum_{i=1}^{r} i^{-1}$

For large $r$ this formula can be simplified to

$t \approx r^{2} \log r$.

2b They are no longer involved in the search process, which improves the efficiency to a mean number of testing events

$t=r(r+1) / 2$.

For large $r$ this formula can be simplified to

$t \approx r^{2} / 2$.

\section{Systematic homologue search}

With a systematic homologue search strategy repetition of unsuccessful testing events is avoided. It invokes a mechanism to ensure that each possible combination of recognition sites is tested only once. This process would require a high degree of (spatial) organization within the nucleus. Its simplest variant could be modelled best as the slithering along each other of chromosomes arranged in a flattened ring or chain (Fig. 3). Several scenarios are conceivable.

3a First, each possible pair of recognition sites is involved in a testing event exactly once. Homologues which have found each other continue to participate in the search. The process comes to an end when all possible combinations have been tested. The precise number of testing events would be

$t=r(2 r-1)$.
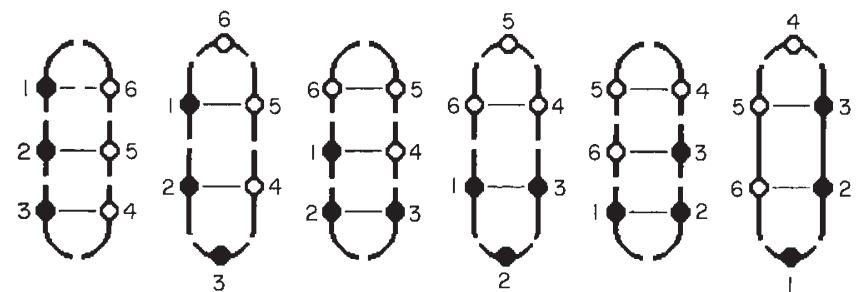

Fig. 3 Simplistic representation of an arrangement of chromosomes which would satisfy the requirements of the systematic homologue search model variant $3 a$. Homology recognition sites arranged in a flattened ring pass by each other once during a half-turn of the ring. Their sequence within the ring is not essential. For further explanations see legend to Fig. 1.
For large $r$ this formula can be simplified to

$t \approx 2 r^{2}$.

3b Systematic homologue search, too, can be restricted to search of the complementary genome only, as expounded in model 2. Each chromosome of one parental set contacts the chromosomes of the other set only. In this case, two chromosome chains/rings, made of a parental set each, would move relative to each other, conferring proximity to any pair of allelic sites only once in such a cycle. Here, the number of testing events would be reduced to

$t=r^{2}$.

By a formal mathematical approach several additional variants of the systematical search model were constructed ( $c f$. Appendix II). However, they are difficult to translate into a meaningful cytological equivalent, i.e., a very sophisticated machinery would have to be invoked to support the search processes. We want to expound only the following variants which rate best of all search strategies mathematically treated in Appendix II.

3c One chromosome screens the others systematically until it finds its homologue. The next chromosome screens the remaining chromosomes for homology, except for the two that have already come together before. The process ends when each chromosome has found its homologue. This might happen long before all possible combinations have been tested. In this case, the average number of testing events is

$t=r(r+1) / 2$.

For large $r$ this formula can be simplified to $t \approx r^{2} / 2$.

3d Also for this variant there exists the additional option of exclusive intergenomic search which brings the mean number of testing events down to

$t=r(r+3) / 4$.

For large $r$ this formula can be simplified to $t \approx r^{2} / 4$.

\section{Limited homologue search}

Some chromosome pairs may be - due to spatial predisposition - more likely to engage in mutual testing than others. If there exists some degree of order in the nucleus, then it is a matter of only limited homologue search to rearrange the predisposed chromosomes properly or to pair the remaining chromosomes which do not benefit from predisposition directly. 
The two extreme variants of this model are: (i) the mean number of testing events is the same as in model 1 with a chromosome number diminished by two. This would be the case if one chromosome pair would always come together automatically. This could be easily imagined of a nucleolus organizing pair which is linked by a common nucleolus; (ii) only one testing event would be necessary in the case of ideal Ashleychains (see Ashley, 1979). This would apply if the chromosomes of both parental genomes were arranged in two identical chains, the pair as a whole starting from a single point (see below).

\section{Discussion}

\section{Appraisal of models of homologue search in the light of experimental evidence}

Search for homology can be envisaged as being random, i.e. occurring in the course of accidental contacts between chromosomes or systematical, i.e. by a screening mechanism. Random search of the whole diploid genome (model 1) ratès worst in terms of expenditure (Fig. 4).

Effort on homologue search would be greatly reduced if chromosomes could discriminate between members of their own parental and the alien set. This would provide for mechanisms for preferential inter-

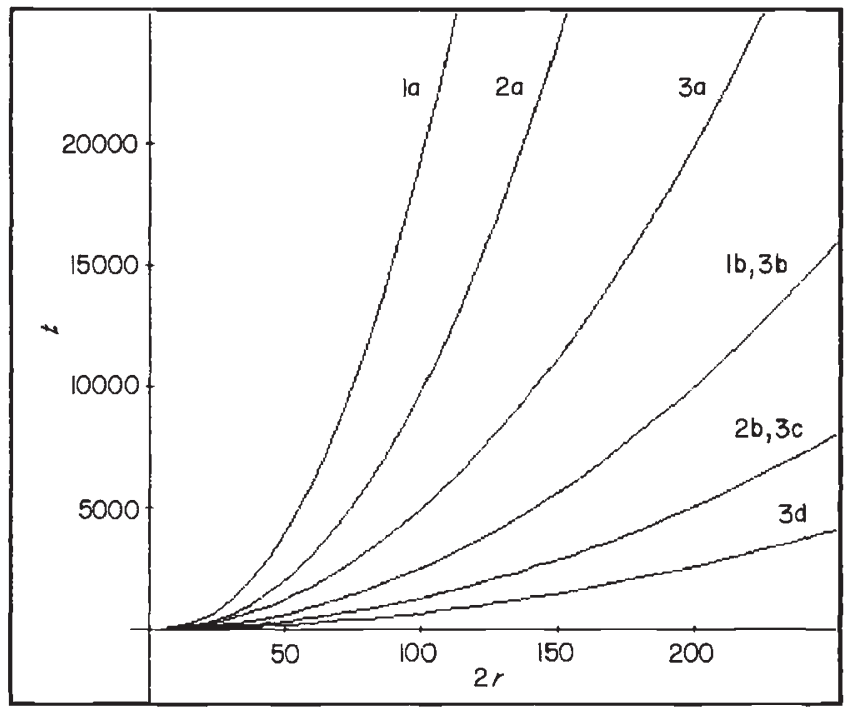

Fig. 4 Graphs of the functions for the (average) number of homology testing events necessary for given numbers of chromosomes as predicted by the models $1 \mathrm{a}, \mathrm{b}, 2 \mathrm{a}, \mathrm{b}$ and $3 \mathrm{a}$, b, c, d. $2 r$ : number of chromosomes and/or autonomous homology recognition sites, $t$ (mean) number of testing events. [To simplify computation, $\Sigma\left(i^{-1} ; i=1, \ldots, r\right)$ was replaced by $\log r$.] genomic homologue search (model 2). It is conceivable that the two parental genomes in diploids persist as separate entities in some respect. First, they could occupy separate territories in the nucleus. This has been shown for the parental genomes in hybrids (e.g. Brandriff et al., 1991; Leitch et al., 1991; Schwarzacher et al., 1992). It is not known, however, if genome separation will hold true for non-hybrid organisms, too. The two genomes of different parental origin might also differ with respect to their genetic activity by genomic imprinting (Nur, 1990). Genomic imprinting as well as spatial genome separation or a combination of these (see Heslop-Harrison, 1990) could be a criterion by which chromosomes are assigned to the two parental genomes in a diploid, and allow the selective involvement in homologue search with members of the other set only. In terms of expenditure, intergenomic homologue search would seem to be superior to random search, but the model is difficult to reconcile with cytological findings. It is known, e.g. that chromosomes tend to pair preferentially with members of the same parental origin in autotetraploids (Håkansson \& Levan, 1957). Furthermore, chromosomes of one haploid set are capable of pairing and recombining in the absence of a homologous set (Neijzing, 1982), and regularly do so with their sister chromosomes in dihaploids.

A systematic homologue search (model 3) would require a complex sequence of movements of chromosomes relative to each other. One possible process would be the slithering along each other of chromosomes arranged in a flattened ring. Thereby each homology recognition site would make contact with any other (Fig. 3). This process would not depend upon a particular sequence of chromosomes within the ring. This is in contrast with the chains or rings in Ashley's (1979) and Bennett's (e.g. Bennett, 1984) pairing models (see below). Variant 3a of the systematical homologue search model seems to be consistent with the screening of the entire genome by a given sequence in search of homology as has been suggested by Haber et al. (1991) for yeast. They showed that if for a certain DNA tract (the LEU2 locus) there is an allelic as well as several ectopic copies present, gene conversion between the allelic loci is equally frequent as with any of the ectopic loci. They argued that once the $L E U 2$ locus is activated (i.e. competent for active homologue search) it will almost certainly find and interact with one of its allelic or ectopic counterparts. This would mean that any site within the genome is contacted by a searching sequence in the course of meiosis. This is unlikely to be achieved by a series of random contacts but rather suggests that an actively searching sequence passes along one chromosome 
after the other in search of its counterpart. Steele et al. (1991) investigated ectopic gene conversion of ura3 heteroalleles between yeast chromosome III and chromosomes II and V, respectively. They found ectopic gene conversion to be equally frequent in a haploid strain disomic for chromosome III and in an isogenic diploid derivative. In the latter one would expect that the homologous pairing of chromosomes II and V with their respective partners would reduce ectopic recombination with chromosome III. Similarly, one would expect ectopic recombination to be less frequent than allelic recombination in the case reported by Haber et al. (1991). This is because one must assume that along with the reported loci (URA3 and $L E U 2$ ) other loci will be searching for their respective homologues as well. A number of these will be successful in finding homology at allelic sites leading to the alignment of homologous chromosomes. This should enforce a preferential allelic interaction of the URA3 and $L E U 2$ loci.

As this seems not to be the case, there emerges a scenario where a number of loci screen the whole genome in a highly systematic manner and, by and by, these sequences encounter sufficiently homologous tracts and thereby establish connections between homologous chromosomes. At the same time the remaining loci continue to search without interference. This means that if a sequence has already found its homologue, this does not increase the chance of subsequent homologous contacts of other sequences. This, however, would be expected if chromosome pairing following a primary homologous contact promoted homologous contacts of nearby sequences on the same chromosome pair, or if subsequent homologue search were restricted to stretches that had not yet met their homologous counterpart. On the other hand, there remains the possibility that only a few sequences have the capability of homologue recognition, and the $U R A 3$ and LEU2 loci are among them, or these two loci are among the first that enter the search process, well ahead of the rest.

Except for the studies in yeast showing a high incidence of ectopic recombination events even when competing with allelic recombination, which suits the simplest model variant $3 \mathrm{a}$ of the systematical search model, evidence in favour of this model is lacking. The more efficient systematical search strategies (model variants $3 b-3 d$ ) would require an even more sophisticated machinery. However, there is no cytological evidence for the highly ordered structures that would have to be postulated to satisfy these models.

The number of testing events could also be reduced if they did not involve all chromosomes of a set with the same probability. Such limited homologue search (model 4) could apply if the distribution of chromo- somes within the nucleus were non-random. Some order of the chromosomes according to size or centromere position by spatial constraints in the nucleus (e.g. by allocating to some chromosomes more peripheral positions than to others) could make homologues more likely to be preferentially involved in mutual testing events (see Loidl, 1990). Even the fusion of nucleoli, which is a common phenomenon, or some other slight deviation from random chromosome positioning could have a small but significant effect in this respect. The extreme case of this would be a purely mechanistical one-by-one affiliation of homologous chromosomes. A pairing mechanism based on a fixed order of chromosomes within each parental set was proposed by Ashley (1979) and Bennett (1984). If these two sets, each organized as a chain, would align, then each chromosome would automatically match with its homologue at the identical position within the other chain. In theory this could bring down the number of necessary testing events to one anywhere within the chains from where the homologues then would start lining up. However, this model was refuted by Maguire (1983) on the grounds that if the order of chromosomes within one of these chains would be altered, e.g. by a translocation, the consequence for pairing of the complete set would be devastating; observations do not conform with this prediction, though.

Homology testing at meiotic prophase would be dispensable altogether if there existed a mechanism which ensures that only homologous chromosomes are spatially disposed for pairing. This might be the case with intimate somatic homologous pairing (for discussion see Loidl, 1990). This strategy would pay off if somatic homologue pairing (which may be costly to maintain) would be required for proper function of the cell anyway (e.g. for transvection, i.e. the effect of somatic pairing on gene expression - see, e.g. Tartof \& Henikoff, 1991). However, only for diptera is there convincing evidence for somatic pairing of homologues (see, e.g. Loidl, 1990).

In Fig. 4 the numbers of testing events required with different numbers of homology recognition sites are compared for various models.

\section{Strategies to reduce effort on homologue searching}

In addition to non-random search strategies expounded in the previous chapters, there could be conceived other means by which homologue search might be facilitated and the effort be reduced. There are the well known phenomena of general chromosome clustering (i.e. the bouquet and the synizetic knot) and the membrane attachment of telomeres (for discussion see Loidl, 1990). Chromosome clustering does 
not contribute to reducing the number of necessary testing events as calculated above for the various models. However, it can reduce distances between chromosomes in general and hence the movements that are necessary to establish contacts between potential homology recognition sites.

Chromosome numbers in eukaryotes range from $2 n=2$ in the ant Myrmecia pilosa and in the germ-line cells of the threadworm Parascaris univalens to over 700 in a fern (for literature see Johnson et al., 1989). The high numbers reported for plants must be considered to be due to polyploidy and the organisms carrying them are unlikely to be capable of functional meiosis. Therefore, a better estimate for the upper range of chromosome numbers that can be coped with by pairing and segregation comes from mammals. There, the largest known chromosome number, from a rodent, Tympanoctomys barrerae, is $2 n=102$ (Contreras et al., 1990). Thus, based on the assumption of only one pairing initiation event per bivalent, the number of homology recognition sites is $102(2 r)$. If initiation sites of the synaptonemal complex represent homology recognition sites, the figure is even higher. Up to 170 synaptonemal complex initiation sites per cell have been found in Lilium (Gillies et al., 1990); this would correspond with $2 r=340$ homology recognition sites. However, it is uncertain if these act autonomously. In $S$. cerevisiae, Haber et al. (1991) have shown for a $2.2 \mathrm{~kb} L E U 2$ segment that it can be subject to ectopic gene conversion. Assuming that gene conversions at meiosis indicate homology testing events, we may speculate that any DNA segment of appropriate length can serve in homology testing, unless the LEU2 segment happens to contain a specialized recognition site. The diploid yeast genome can be estimated to accommodate about 13,000 segments by and/or at which homology can be tested. The LEU2 locus was interpreted as activated, i.e. involved in the search as the active part in 8 per cent of meioses. If a similar fre- quency of activation would hold for the other segments also, then about 1040 sites would be potential homology recognition sites. Thus either the meiotic machinery can cope with a correspondingly high number of testing events or we must assume that the number of actively searching loci is lower and the LEU2 locus is not representative as its activation is above the average, i.e. it is one of only a few specialized sequences that are capable of homology recognition. Based on the above cytological evidence a number around 200 autonomous homology recognition sites seems a good estimate for the upper limit in higher eukaryotes. In Table 1, required numbers of testing events as predicted by different models are given for some selected values of $2 r$.

In the course of a testing event two non-sister DNA molecules are matched base pair by base pair. This probably requires complex molecular events like strand separation, and perhaps even strand cutting and rehealing for every single testing event. In the extreme case a testing event would imply close spatial contact and systematical matching of any two sequences that come into contact and the screening of hundredthousands of kilobases in order to encounter a homologous sequence. As this must be a very costly process it would be sensible to expect that there exist strategies that keep the number of necessary testing events low. This could be achieved by some elaborate search strategies which reduce the regime of accident on homologue encounter and hence the effort spent on homology search (Fig. 4). However, it seems clear that it must be costly to maintain the complex structures and processes that are necessary to support these sophisticated homology search strategies. Most organisms might therefore have limited themselves to optimizing the random search strategy.

Our calculations have identified two particularly advantageous conditions for efficient search, for which hence no unknown structures or processes have to be

Table 1 Values for the (mean) numbers of testing events $t$ according to different models of homologue search (1a, 1b, 2a, 2b, 3a, $3 \mathrm{~b}, 3 \mathrm{c}, 3 \mathrm{~d}-$ see text) depending on different numbers of chromosomes and/or autonomous recognition sites $2 r$. Selected values for $2 r$ are $2 r=2$ for the lowest possible number of recognition sites in Myrmecia pilosa $(2 n=2), 2 r=46$ for the lowest possible number of recognition sites in man $(2 n=46), 2 r=340$ and $2 r=1040$ for estimates of recognition sites in Lilium and Saccharomyces cerevisiae (see text)

\begin{tabular}{|c|c|c|c|c|c|c|c|c|}
\hline \multirow[b]{2}{*}{$2 r$} & \multicolumn{8}{|l|}{ Models } \\
\hline & $1 \mathrm{a}$ & $1 b$ & $2 a$ & $2 b$ & $3 a$ & $3 b$ & $3 c$ & $3 d$ \\
\hline 2 & 1 & 1 & 1 & 1 & 1 & 1 & 1 & 1 \\
\hline 46 & 3865 & 529 & 1975 & 276 & 1035 & 529 & 276 & 150 \\
\hline 340 & 329,410 & 28,900 & 165,191 & 14,535 & 57,630 & 28,900 & 14,535 & 7353 \\
\hline 1040 & $3,691,196$ & 270,400 & $1,847,374$ & 135,460 & 540,280 & 270,400 & 135,460 & 67,990 \\
\hline
\end{tabular}


invoked. One is that homology recognition sites that have found their partner do not continue in the ongoing homology search; hence model variants $1 \mathrm{~b}, 2 \mathrm{~b}$ and $3 c$ are superior to variants $1 \mathrm{a}, 2 \mathrm{a}$ and $3 \mathrm{a}$, respectively (Fig. 4). (This is not as self-evident as it may seem because two homologous sequences could make contact and even initiate a synaptonemal complex and nevertheless continue molecular matching processes with other recognition sequences!) The other advantageous condition is that tests at non-homologous sites are not repeated. This could be best achieved by a systematical search strategy (model 3 ), but also by a concerted action of recognition sites within one and the same chromosome. So it would beneficial for chromosomes with more than one potential recognition site if, as soon as the first has made contact with its homologous counterpart, the remaining recognition sites no longer act independently but become primarily devoted to testing homology in adjacent regions of the same two chromosomes (which may be simply for sterical reasons). Only if homology is not found there (as would be the case with reciprocal translocations) does the testing process extend to other chromosomes. One could speculate that it is the synaptonemal complex which, by virtue of its zipper-like growth, carries the signal reporting a successful homologous encounter along a pair of homologues and thus suppresses superfluous testing events.

\section{Acknowledgements}

We are grateful to Dr G. Karigl for plotting the functions for Fig. 4 and to Dr J. S. Heslop-Harrison for valuable comments on the manuscript. This work was supported by grant no. P7843 of the Austrian Science Foundation to J. L.

\section{References}

ASHLEY, T. 1979. Specific end-to-end attachment of chromosomes in Ornithogalum virens. J. Cell Sci., 38, 357-367.

BENNETT, M. D. 1984. Premeiotic events and meiotic chromosome pairing. Symp. Soc. Exp. Biol., 38, 87-121.

BRANDRIFF, B. F., GORDON, L. A., SEGRAVES, R. AND PINKEL, D. 1991. The male-derived genome after sperm-egg fusion: spatial distribution of chromosomal DNA and paternal-maternal genomic association. Chromosoma, 100, 262-266.

CARPENTER, A. T. C. 1987. Gene conversion, recombination nodules, and the initiation of meiotic synapsis. BioEssays, 6, 232-236.

CONTRERAS, L. C., TORRES-MURA, J. C. AND SPOTORNO, A. E. 1990.

The largest known chromosome number for a mammal, in a South American desert rodent. Experientia, 46, 506-508.
GILliES, C. B., DOLlin, A. E. AND DAI, K. 1990. Chromosomal and genetic factors influencing synaptonemal complex formation. Chromosomes Today, 10, 297-310.

HABER, J. E., LEUNG, W.-Y., BORTS, R. H. AND LICHTEN, M. 1991. The frequency of meiotic recombination in yeast is independent of the number and position of homologous donor sequences: implications for chromosome pairing. Proc. Natl. Acad. Sci. U.S.A., 88, 1120-1124.

HÅKANSSON, A. AND LEVAN, A. 1957. Endo-duplicational meiosis in Allium odorum. Hereditas, 43, 179-200.

HESLOP-HARRISON, J. S. 1990 . Gene expression and parental dominance in hybrid plants. Development (Suppl.), 21-28.

JOHNSON, M. A. T., KENTON, A. Y., BENNETT, M. D. AND BRANDHAM, P. E. 1989. Voanioala gerardii has the highest known chromosome number in the monocotyledons. Genome, 32, 328-333.

LEITCH, A. R., SCHWARZACHER, T., MOSGÖLLER, W., BENNETT, M. D. AND HESLOP-HARRISON, J. S. 1991. Parental genomes are separated throughout the cell cycle in a plant hybrid. Chromosoma, 101, 206-213.

LiCHTEN, M., GOYON, C., SCHULTES, N. P., TRECO, D., SZOSTAK, J. W., HABER, J. E. AND NICOLAS, A. 1990. Detection of heteroduplex DNA molecules among the products of Saccharomyces cerevisiae meiosis. Proc. Natl. Acad. Sci. U.S.A., 87, 7653-7657.

LOIDL, J. 1990. The initiation of meiotic chromosome pairing: the cytological view. Genome, 33, 759-778.

MAGUIRE, M. P. 1983. Homologous chromosome pairing remains an unsolved problem: a test of a popular hypothesis utilizing maize meiosis. Genetics, 104, 173-179.

NEIJZING, M. G. 1982. Chiasma formation in duplicated segments of the haploid rye genome. Chromosoma, $\mathbf{8 5}$, 287-298.

NUR, U. 1990. Heterochromatization and euchromatization of whole genomes in scale insects (Coccoidea: Homoptera). Development (Suppl.), 29-34.

PADMORE, R., CAO, L. AND KLECKNER, N. 1991. Temporal comparison of recombination and synaptonemal complex formation during meiosis in $S$. cerevisiae. Cell, 66, 1239-1256.

SCHWARZACHER, T., HESLOP-HARRISON, J. S., ANAMTHAWAT-JONSSON, K., FINCH, R. A. AND BENNETT, M. D. 1992. Parental genome separation in reconstructions of somatic and premeiotic metaphases of Hordeum vulgare $\times H$. bulbosum. J. Cell Sci., 101, 13-24.

SMITHIES, O. AND POWERS, P. A. 1986. Gene conversions and their relation to homologous chromosome pairing. Phil. Trans. R. Soc. Lond., B 312, 291-302.

STEELE, D. F., MORRIS, M. E. AND JINKS-ROBERTSON, S. 1991. Allelic and ectopic interactions in recombination-defective yeast strains. Genetics, 127, 53-60.

SUN, H., TRECO, D., SCHULTES, N. P. AND SZOSTAK, J. W. 1989. Double-strand breaks at an initiation site for meiotic gene conversion. Nature, 338, 87-90.

TARTOF, K. D. AND HENIKOFF, S. 1991. Trans-sensing effects from Drosophila to humans. Cell, 65, 201-203. 


\section{Appendix}

\section{Derivation of formulae (1) to (8)}

The validity of (5) and (6) is evident. For the proof of the formulae (1) to (4), (7) and (8) the following formulae are needed:

$\sum_{i=1}^{n}\left(\begin{array}{l}n \\ i\end{array}\right)(-1)^{i} i^{-1}=-\sum_{i=1}^{n} i^{-1}$

$\sum_{i=1}^{n}\left(\begin{array}{c}n \\ i\end{array}\right)(-1)^{i} i^{0}=-1$

$\sum_{i=1}^{n}\left(\begin{array}{c}n \\ i\end{array}\right)(-1)^{i} i^{m}=0$

$\sum_{i=1}^{n}\left(\begin{array}{l}n \\ i\end{array}\right)(-1)^{i} i^{n}=(-1)^{n} n !$

$\sum_{i=1}^{n}\left(\begin{array}{c}n \\ i\end{array}\right)(-1)^{i} i^{n+1}=(-1)^{n} n(n+1) ! / 2$

$b_{k+1, s}=\sum_{i=1}^{k+1} b_{k, s-i}$

$c_{k+1, s}=\sum_{i=1}^{2 k+1} c_{k, s-i}$

$\sum_{k=n}^{\infty} k x^{k-1}=\left[n x^{n-1}(1-x)+x^{n}\right] /(1-x)^{2}$

$\left|\bigcup_{i=1}^{n} A_{i}\right|=\sum_{\phi \neq I \subseteq\{1, \ldots, n\}}(-1)^{|I|+1}\left|\bigcap_{i I} A_{i}\right|$

$\sum_{\substack{i_{1}, \ldots, i_{i} \geq 0 \\ i_{1}+\ldots+i_{k}=n}} \prod_{j=1}^{k} a_{j}^{i j}=\sum_{j=1}^{k} a_{j}^{n+k-1} \prod_{\substack{s=1 \\ s \neq j}}^{k}\left(a_{j-a s}\right)^{-1}$.

Here $n$ and $k$ are positive integers, $m \in\{1, \ldots, n-1\}, s$ is an integer, $b_{k s}$ :

$=\left|\left\{\left(i_{1}, \ldots, i_{k}\right) \in \prod_{j=1}^{k}\{1, \ldots, j\} \mid i_{1}+\ldots+i_{k}=s\right\}\right|$,

$c_{k s}:$

$=\left|\left\{\left(i_{1}, \ldots, i_{k}\right) \in \prod_{j=1}^{k}\{1, \ldots, 2 j-1\} \mid i_{1}+\ldots+i_{k}=s\right\}\right|$,

$x$ is a real number with $|x|<1, A_{1}, \ldots, A_{n}$ are finite sets and $a_{1}, \ldots, a_{k}$ are mutually distinct positive real numbers. (Here and in the following $0^{0}$ is defined to be 1.)

(9) follows from

$\sum_{i=1}^{n+1}\left(\begin{array}{c}n+1 \\ i\end{array}\right)(-1)^{i} i^{-1}-\sum_{i=1}^{n}\left(\begin{array}{c}n \\ i\end{array}\right)(-1)^{i} i^{-1}=-1 /(n+1)$. 
(10)-(13) follow by evaluating both sides and the first $n+1$ derivatives of both sides of

$\sum_{i=0}^{n}\left(\begin{array}{l}n \\ i\end{array}\right) x^{i}=(x+1)^{n}$ at $x=-1$.

(14) and (15) are evident. (16) follows by differentiating both sides of

$\sum_{k=n}^{\infty} x^{k}=x^{n} /(1-x)$

(17) can be proved by induction on $n$.

In order to prove (18) we consider the generating function of the left side of (18) and use the decomposition into partial fractions and the 'limit method'. This yields

$$
\begin{aligned}
& \sum_{n=0}^{\infty} x^{n} \sum_{\substack{i_{1}, \ldots, i_{k} \geq 0 \\
i_{1}+\ldots+i_{k}=n}} \prod_{j=1}^{k} a_{j}^{i j}=\sum_{i_{1}, \ldots, i_{k} \geq 0} \prod_{j=1}^{k}\left(a_{j} x\right)^{i j}=\prod_{j=1}^{k} \sum_{s=0}^{\infty}\left(a_{j} x\right)^{s}=\prod_{j=1}^{k}\left(1-a_{j} x\right)^{-1}=\sum_{j=1}^{k}\left(1-a_{j} x\right)^{-1} \prod_{\substack{s=1 \\
s \neq j}}^{k}\left(1-a_{s} / a_{j}\right)^{-1} \\
& =\sum_{j=1}^{k} a_{j}^{k-1} \sum_{n=0}^{\infty} a_{j}^{n} x^{n} \prod_{\substack{s=1 \\
s \neq j}}^{k}\left(a_{j}-a_{s}\right)^{-1}=\sum_{n=0}^{\infty} x^{n} \sum_{j=1}^{k} a_{j}^{n+k-1} \prod_{\substack{s=1 \\
s \neq j \\
s \neq j}}^{k}\left(a_{j}-a_{s}\right)^{-1}
\end{aligned}
$$

completing the proof of $(18)$.

Now let $T$ denote the total number of testing events which occur until all chromosomes have found their respective partner. Then in the various cases we obtain the following formulae for the probability that $T$ has the value $k$ :

$$
\begin{aligned}
& r\left(\begin{array}{c}
2 r \\
2
\end{array}\right)^{-k} \sum_{j=0}^{r-1}\left(\begin{array}{c}
r-1 \\
j
\end{array}\right)(-1)^{j}\left[\left(\begin{array}{l}
2 r \\
2
\end{array}\right)-j-1\right]^{k-1} \quad(k \geq r) \\
& \sum_{\substack{i_{1}, \ldots, i_{r-1} \geq 0 \\
i_{1}+\ldots+i_{r-1}=k-r}} \prod_{j=1}^{r-1}\left\{\left[1-(j+1) /\left({ }^{2 j+2}\right)\right]^{i} j(j+1) /\left({ }_{2}^{2 j+2}\right)\right\} \quad(k \geq r) \\
& r^{-2 k+1} \sum_{j=0}^{r-1}\left(\begin{array}{c}
r-1 \\
j
\end{array}\right)(-1)^{j}\left(r^{2}-j-1\right)^{k-1} \quad(k \geq r) \\
& \sum_{\substack{i_{1}, \ldots, i_{r-1} \geq 0 \\
i_{1}+\ldots+i_{r-1}=k-r}} \prod_{j=1}^{r-1}\left\{\left[1-(j+1) /(j+1)^{2}\right]^{i} j(j+1) /(j+1)^{2}\right\} \quad(k \geq r) \\
& \left.\sum_{\substack{i_{1}, \ldots, i_{r} \geq 1 \\
i_{1}+\ldots+i_{r}=k \\
i_{1} \leq 3}} \prod_{j=1}^{r} \llbracket\left\{\prod_{s=1}^{i_{j}-1}[(2 j-s-1) /(2 j-s)]\right\}\left(2 j-i_{j}\right)^{-1}\right] \quad\left(r \leq k \leq r^{2}\right) \\
& \left.\sum_{\substack{i_{1}, \ldots, i_{r} \geq 1 \\
i_{1}+\ldots+i_{1}=k \\
i_{1} \leq 1, i_{2} \leq 2, \ldots, i_{s} \leq r}} \prod_{j=1}^{r} \llbracket\left\{\prod_{s=1}^{i_{j}-1}[(j-s) /(j-s+1)]\right\}\left(j-i_{j}+1\right)^{-1}\right] \quad[r \leq k \leq r(r+1) / 2] .
\end{aligned}
$$

Now (1) to (4), (7) and (8) follow after some calculations.

\section{A mathematical classification of theoretically possible cases}

Consider the following model assumptions:

$i=1$ : there is a diploid set of $2 r$ chromosomes (autonomous recognition sites) containing $r$ different ones, two of each kind; 
$i=2$ : the two parental sets of chromosomes (autonomous recognition sites) constitute separate pools each of which contains $r$ different chromosomes (autonomous recognition sites);

$j=1$ : two chromosomes are taken simultaneously from the diploid set (one out of each parental set) and are tested. If the two chromosomes are homologous then they are linked. Otherwise they again join the diploid set (their respective parental sets) and again two homologous chromosomes of the diploid set (one out of each parental set) meet, and so on;

$j=2$ : a chromosome is taken from the diploid set (from the first parental set). Then a second chromosome is taken from the diploid set (from the other parental set) and is tested by the first. If they are homologous then they are linked. Otherwise the second chromosome is returned to the diploid set (to its parental set) and again a second chromosome is taken from the diploid set (from the second parental set) and tested, and so on;

$j=3$ : a chromosome is taken from the diploid set (from the first parental set). Then a second chromosome is taken from the diploid set (from the other parental set) and is tested by the first. If they are not homologous then the second chromosome is put aside and another second chromosome is taken from the diploid set (from the second parental set) and tested and so on, until a homologous chromosome is found. Then the homologous chromosomes are linked and the chromosomes which were put aside are returned to the diploid set (to the second parental set);

$k=1$ : linked chromosomes continue to be involved in the search process;

$k=2$ : linked chromosomes no longer participate in the search process; it should be remarked that all choices are understood to be at random.

For $1 \leq i \leq 2,1 \leq j \leq 3$ and $1 \leq k \leq 2$ let $(i, j, k)$ denote the corresponding model. Then model 1 a (formula 1 ) corresponds to $(1,1,1)$, model $1 \mathrm{~b}$ (formula 2 ) corresponds to $(1,1,2)$, model $2 \mathrm{a}$ (formula 3 ) corresponds to $(2,1,1)$, model $2 \mathrm{~b}$ (formula 4 ) corresponds to $(2,1,2)$, model $3 \mathrm{c}$ (formula 7 ) corresponds to $(1,3,2)$ and model $3 \mathrm{~d}$ (formula 8 ) corresponds to $(2,3,2)$. With the above notation one obtains for the probability that $T$ assumes the value $k$ in the case of the model $(1,2,2)$

$$
\sum_{\substack{i_{1}, \ldots, i_{r-1} \geq 0 \\ i_{1}+\ldots+i_{r-1}=k-r}} \prod_{j=1}^{r-1}\left\{\left[1-(2 j+1)^{-1}\right]^{i j}(2 j+1)^{-1}\right\} \quad(k \geq r)
$$

and in the case of the model $(2,2,2)$

$$
\sum_{\substack{i_{1}, \ldots, i_{r-1} \geq 0 \\ i_{1}+\ldots+i_{r-1}=k-r}} \prod_{j=1}^{r-1}\left\{\left[1-(j+1)^{-1}\right]^{i j}(j+1)^{-1}\right\} \quad(k \geq r) .
$$

(In the cases $j=2$ and $j=3$ only the number of the choices of the second chromosome is counted.)

Now it is not hard to see that the probability distribution of $T$ in $(1,1,2)$ is the same as in $(1,2,2)$ and that this distribution in $(2,1,2)$ is the same as in $(2,2,2)$. So in eight of the possible 12 cases for $(i, j, k)$ a formula for the expected value of $T$ could be derived. Six of these correspond to the models $1 \mathrm{a}, 1 \mathrm{~b}, 2 \mathrm{a}, 2 \mathrm{~b}, 3 \mathrm{c}$ and $3 \mathrm{~d}$. [Models $3 \mathrm{a}$ and $3 \mathrm{~b}$ with formulas $(5)$ and $(6)$ do not fit into the classification scheme $(i, j, k)$.] Considering all these eight models one makes the following observation: by passing from $i=1$ to $i=2$ efficiency of the search algorithm is raised by a factor 2 , by passing from $j=1$ to $j=2$ the efficiency does not change and by passing from $j=2$ to $j=3$ the efficiency is again raised by a factor 2 . Finally, by passing from $k=1$ to $k=2$ the efficiency is raised by a factor $2 \log r$.

Assuming that these changes in the efficiency are valid for all 12 cases, one obtains the following list including the triple $(i, j, k)$, the number of the corresponding formula within the text, the exact value of the expectation of $T$ and the approximate value of the expectation of $T$.

(1)

$$
r(2 r-1) \sum_{i=1}^{r} i^{-1} \quad 2 r^{2} \log r
$$

(2) $r^{2}$

$r^{2}$

$2 r^{2} \log r$

$r^{2}$

$r^{2} \log r$

(7) $r(r+1) / 2$

$r^{2} / 2$
(3)

$$
r^{2} \sum_{i=1}^{r} i^{-1}
$$

$$
r(r+1) / 2
$$$$
r^{2} / 2
$$

$$
r(r+1) / 2
$$$$
r^{2} \log r
$$

$$
r(r+3) / 4
$$$$
r^{2} / 2
$$

$r^{2} \log r$

$r^{2} / 2$

$r^{2} / 2$

$\left(r^{2} / 2\right) \log r$

$r^{2} / 4$. 